\title{
El móvil como herramienta para el perfil del nuevo periodista
}

\author{
Ma Belén Andueza LóPez \\ Universidad Antonio de Nebrija \\ mandueza@nebrija.es \\ Rosa Pérez Arozamena \\ Universidad Antonio de Nebrija \\ rosaparozamena@gmail.com
}

\begin{abstract}
Resumen:
Impartir una asignatura denominada Taller de Tecnologías Informativas, requiere por parte del profesorado una innovación permanente y un estado de alerta ante los adelantos tecnológicos que afectan al periodismo. Bajo esta premisa decidimos realizar una experiencia denominada Imaginación al poder con el fin de que los alumnos utilizasen sus móviles como herramienta para la grabación de piezas informativas. Si vivimos en la Era de Internet, enseñemos desde la Era de Internet..
\end{abstract}

Palabras clave: TIC; Docencia; Innovación; Formas; Móviles.

The mobile phone as a tool for the profile of the new journalist

\begin{abstract}
:
Teaching a subject called Informational Technologies Workshop, by teachers, requires a constant innovation and alertness to the technological advances that affect journalism. Under this premise, we decided to conduct an experiment called Imagination to power so that students used their mobiles as a tool for recording pieces of information. If we live in the Internet Age, teach from the Internet Age.
\end{abstract}

Key Words:_TIC; Teaching; Innovation; Forms; Mobile phones.

\section{Referencia normalizada:}

Andueza López, M.B. y Pérez Arozamena, R. (2014): El móvil como herramienta para el perfil del nuevo periodista. Historia y Comunicación Social. Vol. 19. Núm. Especial Enero. Págs. 591-602.

Sumario: 1. Introducción. 2. Metodología. 3. Imaginación al poder. 3.1. Las TIC. 3.2. Nuestros ganadores. 4. El nuevo periodista. 5. Conclusiones. 6. Referencias bibliográficas. 6.1 Libros. 6.2. Capítulos libros. 6.3. Publicaciones web. 7. Anexos

\section{Introducción}

Decir que nos encontramos en plena Era de Internet resulta casi anticuado. Vivimos un momento de cambio, en el que la comunicación 2.0 parece ya superada y sustituida por la 3.0. Las redes sociales, el Whatsapp, las aplicaciones, así como las tablets y los smartphones han cambiado la forma en la que nos relacionamos con los 
demás e, incluso, y más interesante para nosotros como profesionales de la comunicación, la forma en la que nos comunicamos. Es por ello, que como docentes, en trato con el sector más relacionado con este tipo de tecnologías: el $40 \%$ de los jóvenes españoles afirma que no puede vivir sin su móvil, habremos de ser conscientes de su importancia, de su potencial uso, así como de sus valores añadidos. De este modo, adaptarse a esta realidad de la sociedad 2.0, pasa por la explotación de las tecnologías de la información y la comunicación en los procesos de formación universitarios, y es que los centros de educación superior, como parte puntera de la sociedad no pueden quedarse al margen de los cambios que acaecen como una institución inmovilista y a parte de la sociedad.

Así bien, decidimos poner en marcha una experiencia novedosa que incluyese el uso del móvil. Si no pueden separarse de sus teléfonos ni en clase, aprovechemos esta situación. Creímos que el escenario ideal para llevar a cabo este experimento era la clase de la asignatura Taller de Tecnologías Informativas que se imparte en los planes de Periodismo. Bajo el epígrafe Imaginación al poder propusimos a los alumnos grabar una pieza de tema libre para un informativo de televisión cuyo principal requisito era que fuese grabada con un dispositivo móvil. Asimismo, para aquellos que aún no disponen de un smartphone, planteamos que lo hiciesen con una cámara fotográfica digital.

Imaginación... no es más que la excusa para ver trabajar con las TIC. Las nuevas tecnologías son una parte fundamental en la vida de los jóvenes universitarios: teléfonos móviles de última generación y cámaras de fotos con mayor calidad que muchas cámaras de vídeo. Al participar en esta experiencia, quisimos que los alumnos de periodismo fueran conscientes de que gracias a las TIC que están a su completo alcance, pueden elaborar una información, en cualquier momento, sin necesidad de contar con los equipos televisivos tradicionales. De este modo, pudimos comprobar cómo las tecnologías impactan en nuestro pensamiento, analizando cómo vemos la realidad y cómo podemos narrarla a través de las TIC, aprovechando todo su potencial.

Desde el punto de vista de la docencia, se pretende no sólo enseñar a los alumnos a utilizar sus dispositivos móviles como herramientas para el desarrollo de su futura profesión, sino que queremos abrir su mente a nueva forma de concebir la realidad que les rodea y que gracias a las TIC puedan ser mejores profesionales por poder contar con los instrumentos necesarios para narrar lo que quieran en el momento que estimen oportuno. Es además, una forma práctica de hacerles llegar la asignatura de Nuevas Tecnologías que en muchas ocasiones, "suele ser infravalorada y víctima de una confusa programación en los planes de estudios" (Benítez, Pueo y Sánchez 2011:659).

Así bien, partiendo de la base de que si vivimos en La Galaxia Internet (Castells, 2002), hemos de enseñar viviendo en dicha galaxia, iniciamos esta experiencia, Imaginación al poder, como un ensayo general del necesario cambio metodológico 
al que han de enfrentarse los estudios en Periodismo para formar a profesionales capaces de desarrollarse en el nuevo campo de la comunicación.

\section{Metodología}

Si partimos de la base que estamos viviendo una época de cambios, en la que las TIC hacen posible una nueva realidad, tendremos, necesariamente, que cambiar también nuestra forma de enseñar. Nuevas realidades han de llevar consigo nuevas formas de hacer y la metodología educativa en las aulas de las universidades no puede ser menos. En este contexto, se producen ciertos cambios en los actuales enfoques de en enseñanza, dominados actualmente por:

1. La importancia de la interactividad en el proceso de aprendizaje.

2. El cambio de rol de profesores de sabio a guía.

3. La necesidad de destrezas de gestión del conocimiento y de habilidades para el trabajo en equipo.

4. Y el movimiento hacia el aprendizaje basado en recursos más que en paquetes.

(Salinas 2004:3).

Tendremos, entonces, que modificar nuestro enfoque metodológico, el cual será entendido según los parámetros que describe Salinas cuando define el entorno de aprendizaje: "espacio o comunidad organizados con el propósito de lograr el aprendizaje" (2004:2). Nuestro nuevo entorno, creado a partir de la experiencia Imaginación al poder, es un modelo en el que el alumno es el centro, desplazando al docente al papel de guía. De este modo, el alumno aprenderá por sí mismo a partir de la formulación de proyectos, en los que el profesor propone un pretexto como elemento de interés -grabación de una pieza para un informativo-. Esta pedagogía incentiva la investigación personal y desarrolla las potencialidades particulares de cada alumno, ya que se basa en el interés de cada uno de ellos y construye el conocimiento a través de la experiencia. Una nueva forma de aprendizaje esbozada ya en los parámetros del EEES y que hemos llevado a cabo como parte de un proceso novedoso de enseñanza, con un cambio en las formas, el cual, por ende, implica un cambio también en los contenidos y en la forma de entender la enseñanza de ciertas asignaturas.

En resumen, nos encontramos ante una nueva perspectiva, abierta gracias a las nuevas tecnologías y el poder que las mismas tienen. Es decir, abrimos una nueva "estrategia didáctica" (Colom, Salinas y Sureda: 1988), que sienta sus bases tanto en métodos, como medios y técnicas. Esta innovación metodológica es una "forma creativa de selección, organización y utilización de los recursos humanos y materiales" (Salinas 2004:4). 
Por último, este cambio metodológico significa aceptar ciertas realidades. El uso de la tecnología no es el fin último de esta experiencia, es tan sólo el vehículo que llevará al alumno al desarrollo personal y académico, tal y como afirman Benítez, Pueo y Sánchez la tecnología es "la herramienta capacitadora que permite hacer realidad los distintos proyectos audiovisuales" (2011: 675). Asimismo, es necesario asumir la importancia de que los nuevos periodistas han de saber manejar medios técnicos, desdeñar esta posibilidad es darle la espalda al actual mercado laboral donde el profesional todoterreno es el más buscado. Por otro lado, y siguiendo la línea abierta por estos investigadores en su artículo Tecnología y pensamiento: un vínculo fundamental en la enseñanza de las tecnologías de los medios audiovisuales, y como hemos mencionado, sin menospreciar el valor del uso de las tecnologías, hemos de ser conscientes de que el uso de las TIC lleva consigo un proceso creativo particular: pensar en su empleo es pensar de un modo distinto, comprendiendo la realidad de forma diferente al resto de ciudadanos no periodistas. Los nuevos profesionales, gracias al cambio metodológico propuesto, serán capaces de ver su entorno como un espacio informativo sin limitaciones espacio-temporales, y es que gracias a sus móviles podrán ser parte de la noticia y narrarla a la vez.

Este cambio en las metodologías empleadas exige un esfuerzo por parte del docente, muchas veces acomodado en su rutina. Somos conscientes de las exigencias que conlleva el puesto de profesor universitario; no sólo las clases pueblan su rutina diaria, sino que hay que dedicar horas a la investigación, al trato con los alumnos, claustros, papeleo... Aun así, queremos poner el acento en este aspecto, animando al profesorado a pensar en nuevas formas que realmente lleguen a conectar con sus alumnos, aprovechando sus filias, sus aficiones, sus gustos personales, como vehículo para llegar hasta ellos.

\section{Imaginación al poder}

A nuestros alumnos se les propuso un reto: realizar una noticia que sería emitida en un programa especial, Nebrija: imaginación al poder. Para este informativo ficticio los alumnos tendrían que realizar una pieza de tema libre, y todos los trabajos remitidos entrarían en un concurso con un premio acorde a la experiencia.

Los alumnos tenían que enviar una introducción, máximo 20", para que el presentador lo leyera a cámara y después cualquier formato utilizado en un noticiario de televisión: directo/falso directo, entrevista, colas, vídeos... Sorprendentemente, y a pesar de la total libertidad que se les dio, todos optaron por el formato más convencional, con una intro+vtr.

A pesar de la total libertad que se les dio en cuanto a la temática y el formato, se establecieron una serie de parámetros:

1. En caso de vídeo, la duración no debía ser mayor de 1:30 segundos. 
2. La grabación debería ser con su dispositivo móvil: teléfono o cámara.

3. En caso de tener editor de vídeo podían montarlo en: Movie Maker, Sony Vegas, Finalcut, Avid...

4. El vídeo debería llevar su off y por supuesto su audio ambiente, incluyendo sí se quería, totales o declaraciones.

5. El formato de vídeo era libre, aunque se recomendaba ".mov/H264".

Lo novedoso del concurso es que se debía grabar y editar ese mismo día, y enviarlo por FTP a Antena 3TV (para ello, se les otorgó un usuario y una contraseña común para poder mandarlo) antes de las 21:00 horas para entrar en el supuesto programa especial. Se valoraría la originalidad, los recursos utilizados y, por supuesto, que el trabajo llegara a tiempo. Aspecto, este último, que se convirtió en determinante, ya que muchos de los trabajos llegaron fuera del plazo establecido.

Y como todo buen trabajo debe tener su premio, establecimos una recompensa muy atractiva a ojos de los alumnos de periodismo. Los ganadores, tres en este caso en concreto, podrían asistir a la preparación del programa Espejo Público, desde las seis de la mañana hasta su finalización, participando en la elaboración y conociendo a todos sus protagonistas: presentadores, realizador, redactores..., e incluso ofreciéndoles la posibilidad de salir a hacer algún directo para el programa.

\subsection{Las TIC}

El conocimiento y adecuado uso de las TIC fue una de las metas de esta experiencia. Especialmente importante era el empleo del móvil para el desarrollo de su futura actividad profesional, pero no era ésta la única tecnología que tuvieron que aprender a manejar, y es que el universo periodístico no se entiende hoy día sin las TIC y los docentes universitarios no podemos dejar de enseñarles a utilizarlas por dos motivos claros. Primero, para dotarles de las herramientas adecuadas, lo que hará de ellos mejores profesionales y más preparados, en segundo lugar.

Hay un acrónimo que cada vez se oye (y utiliza) con mayor frecuencia en todos los medios de comunicación: FTP (siglas en inglés de File Transfer Protocol $=$ Protocolo de Transferencia de Ficheros). Hoy por hoy los correos electrónicos no permiten el envío de ficheros adjuntos de más de 30 megas, y para poder mandar ficheros de mayor peso se requieren otros métodos. El FTP es la opción más empleada para transferencia de archivos y, salvo excepciones, sin límite de megas, entre sistemas conectados a una red TCP (Transmission Control Protocol), denominada o basada en: cliente-servidor. Desde un equipo cliente se puede conectar a un servidor para descargar o enviar archivos.

En un principio esta tecnología tiene enormes ventajas, la principal: es gratis, se utiliza la red mundial de Internet. Otra enorme ventaja, es que podemos conectarnos desde cualquier parte del mundo mediante una red fija, wifi o $3 \mathrm{G}$ (¡ojo la cobertura!). Y, como hemos indicado, sin límite de megas. Obviamente, cuanto mayor es 
el tamaño del fichero, mayor será el tiempo de transferencia y más riesgos de cortes podemos sufrir en el proceso, aunque la transferencia está garantizada. Por otro lado, el FTP tiene también alguna desventaja, ya que está pensado para ofrecer máxima velocidad en la conexión, pero no máxima seguridad, puesto que no hay cifrado, un posible hacker podría capturar los archivos transferidos. Es decir, no hay plena seguridad. Evidentemente cuanto más y mejor organizado y gestionado esté un servidor FTP mejores serán las prestaciones que ofrezca al usuario, desde la velocidad, a la gestión o el tipo de acceso. Actualmente, dada la complejidad y la cantidad de actores que intervienen en la producción televisiva (cadena emisora, productora, delegación, postproductora, agencia de medios, agencia publicitaria, departamentos de marketing, promociones, emisiones...) supone un movimiento continuo de materiales que mediante transferencias por FTP agilizan los procesos y abaratan enormemente los costes de producción.

Mención aparte, pero no menos importante, es todo el proceso posterior de transcodificación de formatos. A la avalancha enorme de formatos y codecs, afortunadamente el mercado responde con sofisticados transcodificadores que resuelven lo que podría llegar a ser un enorme caos de incompatibilidades. En esta misma línea, se encuentran los editores de vídeo. Los móviles más nuevos ya cuentan con editores propios, una maravilla de la tecnología que muchos jóvenes ya manejan con soltura. Además, al tratarse de una pieza de vídeo editada, nuestros alumnos tuvieron que manejar los clásicos editores de vídeo, desde los más profesionales hasta el archiconocido Movie Maker.

No queremos cerrar esta apartado sin hablar de las opciones de transferencia de archivo a través de la nube. Nosotros elegimos el FTP por ser el método empleado en Antena 3TV, televisión vinculada con la Universidad Antonio de Nebrija, y cadena donde se emite el programa que brindaba a los alumnos la oportunidad de colaborar con ellos como premio. Pero, todo lo que sea trabajar con la cloud es altamente interesante, y es que como anunció Steve Jobs hace años: "El centro de la vida digital estará en la nube de Internet". Hay muchas herramientas para el trabajo en la nube, pero dos se están popularizando enormemente ya que nos solucionan satisfactoriamente determinadas necesidades en los medios de comunicación. Éstas son:

1. Wetransfer (www.wetransfer.com). Se caracteriza por su sencillez: acceso vía web y no es necesario estar registrado; la limpieza: diseño de web muy atractivo, con fondos bonitos y nada agresivos; y su precio, es gratis. Nos permite enviar y recibir ficheros de hasta $2 \mathrm{~Gb}$. Lógicamente existe la versión Premium, de pago, que ofrece mayores prestaciones. En las redacciones de noticias, por ejemplo, se sugiere para paliar posibles carencias técnicas de la parte que pretende enviarnos un material (el típico ciudadano que ha grabado con su móvil la riada de su pueblo).

2. A otro nivel se sitúan las empresas que nos ofrecen la posibilidad de enviar, recibir, almacenar y compartir archivos. Por ejemplo los ya conocidos y enor- 
memente populares Dropbox (www.dropbox.com) o Box (www.box.com) que incluso se presentan como alternativa al FTP.

3. Estas opciones están revolucionando la forma de trabajar compartida y el intercambio de materiales, pero lo mejor de todo es que constantemente están buscando la sencillez para evitar el rechazo al cambio. Y todo ello a una velocidad vertiginosa, porque como pasa con las formas personales de comunicación, todas las TIC avanzan diariamente.

\subsection{Nuestros ganadores}

De todos los alumnos participantes, tres fueron los seleccionados debido a la calidad de la grabación, el envío del material dentro del tiempo establecido y la originalidad de la información. Creemos importante reproducir sus propias impresiones, para comprobar cómo vivieron ellos la experiencia y cuáles han sido las enseñanzas que han extraído de la misma, siempre con el ánimo de hacer de ésta una comunicación en la que prime el carácter práctico que tiene que prevalecer en la docencia universitaria.

Irene Santos: "Desde un primer momento supe que quería hacer algo relacionado con las manos, no tenía claro el enfoque concreto, fue saliendo sobre la marcha. ¿Por qué? Pues porque pensé que realmente las manos son muy importantes en nuestro día a día, incluso para expresar emociones y sentimientos. Quería que solo ellas fueran las protagonistas. Nunca tuve un guión bastante definido, sabía que quería mostrar la diversidad de manos existentes y, con ello, las numerosas historias que se esconden tras ellas. Lo que sí que tenía claro es que necesitaba manos de personas ejerciendo su profesión; otras que representaran acciones cotidianas y otras que expresaran sentimientos. Usé mi móvil (sistema Android) en todos los planos, incluso para grabar mi plató y el total de la enfermera. Eso me permitió poder grabar en cualquier momento. La grabación me llevo varios dias porque quería encontrar numerosos planos de manos, y para ello tuve que entrar en tiendas, o pedirle a gente que me iba encontrando por la calle si no les importaba colaborar en mi proyecto. La verdad es que fue divertido ver sus caras de incredulidad cuando se lo proponía.En un primer momento quise editar el proyecto en Avid, cosa que fue imposible porque éste no reconocía los archivos grabados con el móvil. Intenté convertirlos uno a uno, pero como estaba en un pueblo perdido en la sierra de Huelva, la cobertura de Internet era malísima, y no me dejaba. Asi que finalmente opté por editar con el Movie Maker. Pero ahí llegó el segundo inconveniente: ¿cómo editar el sonido ambiente, el off y la música a la vez, cuando este programa sólo tiene un canal de audio? Pues tuve que grabar mi off a la vez que reproducía con el ordenador la música elegida y mientras visionaba los planos que previamente ya había montado. Fue un poco complicado y el resultado no fue el esperado, pero poco más podía hacer. El tercer problema llegó a la hora de mandarlo por Wetransfer (una vez más, la mala cobertura me jugó una mala pasada): el vídeo llegó incompleto”.

Adolfo Izquierdo: "La elección del tema era algo que tenía pendiente hacer desde hace tiempo. Elegí ese tema porque era algo que no llevaba producción y 
que me podría facilitar en el tiempo que teníamos la elaboración del reportaje. Tan solo es necesario ser algo persuasivo para que la gente de la calle y los actores/trabajadores contesten a las preguntas. El reportaje se grabó con una cámara fotográfica reflex con la que se puede obtener gran calidad de imagen. Decidi grabarlo en la tarde por cuestión de compatibilizar horarios con el resto de compromisos, por lo que la grabación de imágenes y entrevistas me llevó unas tres horas aproximadamente. Quería plasmar una gran variedad de trabajos callejeros, pero el día elegido para la grabación tuve la mala suerte de no encontrar ningún músico callejero tan sólo animadores, mimos y actores. En general no tuve ningún problema ni en la grabación ni en las entrevistas ni en el montaje que fue realizado en Avid".

Antonio Hernández: "En un principio, me habia planteado la idea de realizar una noticia relacionada con la festividad de la Constitución Española, pero la imposibilidad de tener acceso a un ejemplar de la Carta Magna me obligó a desechar la idea. Es entonces cuando se me ocurre hacer una noticia relacionada con la Navidad, después de pasear por la calle y ver el contraste que había entre las hojas caducas del suelo y las luces de los árboles navideños, ubicados en la entrada de un hotel. La grabación fue realizada con una cámara fotográfica Fujifilm, de 10 megapixeles. Los planos los obtuve de grabaciones en plena calle, en el interior de tiendas, un centro comercial y en mi domicilio. Los totales se filmaron en una céntrica calle madrileña. Las imágenes se grabaron el mismo día en el que se presentaba el trabajo, con el objetivo de vivir la auténtica experiencia de trabajar en una redacción, en la que te encargan cubrir una información y tienes un tiempo delimitado. No ha existido ningún guión previo. A partir del surgir de la idea, se fue planeando en mi mente una "escaleta", de tal modo que iba grabando aquellas imágenes que consideraba que iban a poder servirme para el off que iba construyendo mentalmente. Posteriormente, el montaje lo realicé en un Sony Vegas de prueba, con bastantes dificultades a la hora de editar, ya que el ordenador se bloqueaba mucho. El montaje, a pesar de ello y, en cuanto a contenido y estética, fue bastante fluido".

\section{El nuevo periodista}

En pleno cambio de contexto comunicativo el perfil de los profesionales de la comunicación ha de evolucionar, así como evoluciona el panorama comunicacional actual, en el que las nuevas tecnologías establecen nuevos parámetros y nuevas vías de comunicación interpersonal y mediática. Nuestro mundo interconectado necesita periodistas formados en las TIC.

Por otra parte, nos encontramos con la crisis económica, la cual ha tenido efectos devastadores en los medios de comunicación. Desde el comienzo de la crisis, en 2008, en España se han destruido 6.393 puestos de trabajo de profesionales de la información y han echado el cierre 197 medios. Por lo que producir información a un 
coste más bajo (tanto en medios técnicos, como en profesionales) será muy interesante para las empresas informativas.

Otra de las patas que configuran el nuevo panorama lo encontramos en los receptores. El mercado actual demanda un nuevo profesional para una nueva sociedad en la que el público ha dejado de ser un ente pasivo y adormecido, para convertirse en una figura que participa, contrasta, critica y que incluso, crea y difunde información propia y original. El máximo exponente de este nuevo perfil de espectador lo encontramos en Twitter, gracias a esta red social, el público sigue la programación minuto a minuto, convirtiéndose en parte de la misma. Gracias a los hashtag, cualquiera puede ser parte de determinado programa de televisión y/o radio. Espectadores activos exigen profesionales igualmente activos, capaces de hacer información en cualquier condición, y es que los antiguos esquemas se han visto superados y el nuevo profesional debe adquirir nuevas herramientas y asumir otras funciones para adaptarse a dicho cambio.

Es por ello, que el periodista de hoy en día ha de reunir una serie de cualidades y conocimientos que le capaciten para ejercer su profesión según la realidad en la que va a desarrollarse. En primer lugar, y como eje vertebrado, el nuevo periodista ha de ser polivalente. Tendrá que ser un profesional capaz de grabar vídeos, montarlos, escribir el guión, contextualizar las historias... Lo que hemos venido a llamar, un periodista todoterreno y versátil. Vinculado a esta idea, encontramos el segundo aspecto fundamental: el multiformato, el nuevo profesional necesita manejarse tanto off como online, siendo consciente de que no es lo mismo crear contenidos para un formato u otro, ni utilizar una TIC que otra. En un mundo en el que estamos sobre informados, será el periodista el que tendrá que ordenar este caos para los ciudadanos, jerarquizando, valorando y dando sentido a este caos informativo. Y por último, no hemos de olvidarnos de la necesidad de profundización, puesto que un profesional de hoy tiene la obligación de explicar el porqué de lo que está pasando. El periodista tendrá que completar la información con todos los recursos que estén a su alcance: vídeos, enlaces, archivos, infografías, noticias relacionadas... aprovechando todo el potencial de los recursos que las nuevas tecnologías nos ofrecen.

Nos encontramos en pleno proceso de cambio. Un cambio continuo y de adaptación de los medios televisivos a una tecnología que no para de crecer. Surgen constantes retos que mantienen despiertos a los departamentos tecnológicos para adaptar un constante avance a un quehacer diario. El nuevo profesional ha de tener este aspecto muy claro en su imaginario, así que tendrá que ser informado de ello desde el momento en el que inicie su formación universitaria. Los docentes les tenemos que transmitir la necesidad del cambio de rol, pasando de ser meros creadores, a ser autores, editores, productores y realizadores de sus informaciones.

El periodista de hoy en día necesita serlo 24 horas, 7 días a la semana, y gracias a los dispositivos móviles podrá ser así, siendo capaces de informar audiovisualmente en un breve espacio de tiempo de lo que es noticia. Atrás está quedando el ir a los lugares donde se produce el acontecimiento, grabar con un operador de cámara, 
volver a la redacción para montar en el ordenador de mesa y enviar a la escaleta la información. Empieza a ser más operativo la grabación con el móvil, tableta o la cámara réflex, como del mismo modo se está evolucionando hacia la edición en estos mismos dispositivos y el lanzamiento a través de FTP como hemos comprobado en esta experiencia. El siguiente paso serán los directos. Dentro de poco tiempo, cuando la calidad mejore, desaparecerá la contratación de satélite para dar paso a un directo, y será a través de Internet como el periodista nos contará lo que está ocurriendo en ese instante. Y es ahí donde el docente tendrá que incidir durante la formación para que nuestros alumnos estén sobradamente preparados.

\section{Conclusiones}

Es innegable que nos encontramos en un momento difícil para la profesión: crisis, cierre de medios, intrusismo... Un momento complicado para aquellos que se encuentran en el mercado laboral, para los que no pueden acceder y para los que aún se encuentran en pleno proceso formativo en las universidades. Como docentes hemos de ser conscientes de la realidad en la que vivimos y de las oportunidades y las amenazas que caracterizan a la presente sociedad multimedia, para que nuestros alumnos salgan lo mejor formados de nuestras aulas. Ayudemos al alumnado formando a profesionales fuertes, capaces de escribir bien, de adaptarse a cada entorno, de ser críticos, y todo ello en un tiempo récord gracias al empleo de las nuevas tecnologías que tienen a su alcance.

Como parte de estas conclusiones y abundando siempre en el hecho de que las TIC son una valiosa herramienta que los nuevos periodistas tienen que saber emplear en su beneficio, hay una idea que no queremos dejar de lado. El periodista multimedia, cuyo perfil hemos esbozado en el apartado anterior, sólo se distinguirá de sus compañeros en los métodos y en las técnicas, pero nunca en los contenidos: la información veraz, rigurosa y honesta al servicio exclusivo de la sociedad, será su fin único. De este modo, las TIC serán una herramienta facilitadora de su trabajo, trabajo que seguirá siendo el mismo: búsqueda de un hecho noticioso, contacto con las fuentes, contraste de información, redacción y publicación. Todo ello mejor, más rápido, más rico y más logrado.

Para concluir queremos animar a todos nuestros compañeros docentes a utilizar su propia imaginación. En un momento en el que nuestro entorno comunicativo cambia día a día, hemos de utilizar nuestra imaginación para poder adaptarnos al mismo. Los profesores universitarios hemos de ser también 2.0 y adaptar nuestras programaciones a la realidad que se impone irremediablemente. Imaginación al poder como base de nuestra innovación docente en contenidos, como sobre todo, en formas que reflejen el poder de las nuevas tecnologías de la información y la comunicación.

Y como no hay mejor conclusión que ver el resultado del trabajo, os invitamos a visitar el blog de la Universidad Nebrija y ver los trabajos ganadores: 
http://blogs.nebrija.es/canalnebrija/2012/12/canalnebrija-especial-navidad/.

\section{Bibliografía}

\subsection{Libros}

APM (2012): Informe Anual de la Profesión Periodística 2012. APM. Madrid.

CASTELLS, Manuel (2002): La Galaxia Internet. Plaza \& Janés. Barcelona.

CEBRIÁN HERREROS, Mariano, FLORES VIVAR, Jesús Miguel (2012): Periodismo en la telefonía móvil. Fragua. Madrid.

CEBRIÁN HERREROS, Mariano (2010): Desarrollos del periodismo en internet. Comunicación Social. Zamora

VV.AA (2004): El comunicador digital. Transformaciones en las rutinas y perfiles profesionales de la comunicación en los nuevos entornos tecnológicos. Universidad Católica S. Antonio Murcia.

VV.AA (2011): Periodismo ciudadano. Fundación Telefónica. Ariel. Madrid.

UNNE (2010): Memoria del Máster de Periodismo en Televisión. Facultad de CC de la Comunicación, Universidad Antonio de Nebrija.

LIBERAL ORMACHEA, Sheila y SIERRA SÁNCHEZ, Javier (2011): Investigaciones educomunicativas en la sociedad multipantalla. Fragua, España.

6.2 Capítulos libros

BENÍTEZ, Anto; PUEO, Basilio y SÁNCHEZ CID, Manuel (2011): Tecnología y pensamiento: un vínculo fundamental en la enseñanza de las tecnologías de los medios audiovisuales en LIBERAL ORMACHEA, Sheila y SIERRA SÁNCHEZ, Javier: Investigaciones educomunicativas en la sociedad multipantalla. Fragua, España.

PERELLÓ, Marc (2011): Communication and education: An approach on how the new information technologies might be changing our methods of knowledge en LIBERAL ORMACHEA, Sheila y SIERRA SÁNCHEZ, Javier: Investigaciones educomunicativas en la sociedad multipantalla. Fragua, España.

SALINAS, Jesús (2004): Innovación docente y uso de las TIC en la enseñanza universitaria en Revista Universidad y Sociedad del Conocimiento. Volumen 1, $\mathrm{n}^{\mathrm{o}} 1$.

SALINAS, Jesús (2004): Cambios metodológicos con las TIC. Estrategias didácticas y entornos virtuales de enseñanza-aprendizaje en Bordón. Volumen 56, números 3 y 4.

6.3 Publicaciones web

ACINAS, Maite (2013): Periodistas del futuro en http://www.empleoyemprendedores.com/2013/04/26/periodistas-del-futuro-un-nuevo-perfil-profesional/. [24-0713] 
BECERRA, Ana Belén (2006): El periodista digital en http://www.dosdoce.com/ articulo/opinion/2780/el-periodista-digital-perfil-de-un-nuevo-comunicador/. [24-07-13]

El País, http://tecnologia.elpais.com/tecnologia/2011/06/06/actualidad/1307350864 850215.html [15-06-2013]

Telefónica http://survey.telefonica.com/ [18-06-2013]

\section{Las autoras}

$\mathrm{M}^{\mathrm{a}}$ Belén Andueza. Doctora por la Facultad de CC de la Información en la Universidad Complutense de Madrid. Acreditada como "profesor doctor de Universidad Privada" por la ACAP. Licenciada en CC de la Comunicación por la Universidad Complutense de Madrid. Licenciada en Sociología por la UNED. Técnico publicista por el Instituto Mirasierra. Título de experto en Avid impartido por el CEV. Profesional de los medios de comunicación como realizadora en TVE y T-5, principalmente en informativos dirigidos por profesionales como Vicente Vallés, Montserrat Domínguez, Juan Pedro Valentín, Ángels Barceló, Hilario Pino, Antonio Lobato... Realizadora de eventos informativos: elecciones legislativas, manifestaciones, conflictos internacionales, Bodas Reales... Elaboración de reportajes educativos para la Comunidad de Madrid. Profesora del Máster de locución y realización televisiva en academias audiovisuales (CEV y CES). Publicaciones de libros y de artículos en revistas del sector sobre comunicación política y realización audiovisual principalmente. Colaboraciones con radios y periódicos de ámbito nacional como Punto Radio, Cadena Ser o Público.

Rosa P. Arozamena. Joven periodista con una fuerte vocación por la docencia. Licenciada en Periodismo por la Universidad de Sevilla y Máster con especialidad en radio y televisión en la Universidad Antonio de Nebrija, siguió con sus estudios en periodismo con el fin de doctorarse. Actualmente, escribe su tesis y se doctorará el año próximo por la Universidad Nebrija. Con el fin de estar altamente cualificada para la enseñanza universitaria, Rosa ha continuado formándose y es Experto en protocolo y comunicación empresarial, pero sobre todo, se ha ido reciclando en temas relacionados con la comunicación 2.0 y las actuales tendencias del mercado, por lo que es Experta en Community Management, posicionamiento en buscadores, SEO, SEM. Asimismo, y como actividad complementaria a sus estudios de doctorado y sus investigaciones en la universidad, ha venido desarrollando su profesión en este mismo campo de la comunicación en Internet, lo cual la acredita como una experta en la materia 2.0. 